\title{
DIFFERENTIAL EQUATIONS AND DIFFERENTIAL CALCULUS IN MONTEL SPACES $\left({ }^{1}\right)$
}

\author{
BY \\ E. DUBINSKY
}

A topological vector space is a set which is equipped with a geometric structure and a topological structure. It is natural to ask if this is sufficient to derive an analytic structure, or if one has to impose it from without as in the case of manifolds. By an analytic structure, we mean the operations of differentiation and integration, and their corresponding properties.

It is well established that integration can be defined reasonably (for a summary of the vast amount of literature on this subject, see Hyers [7]). In the case of differentiation, until recently, little has been done beyond the case of Banach spaces. In the latter case, the program has been completed by Hildebrandt and Graves [6], who developed the calculus, Kerner [8], who characterized the perfect differentials, Michal and Elconin [9], who extended the Picard existence and uniqueness theorems for differential equations, and Dieudonné [4], who showed that the weaker Peano existence theorem, which assumes only continuity, is false for Banach spaces.

To begin the extension to non-normed spaces, Gil de Lamadrid [5], gave a definition of the derivative and started the development of the calculus. In the present paper, for the case of Montel spaces (to be defined below), we extend the calculus, characterize the perfect differentials and prove existence and uniqueness theorems for differential equations.

In this paper, $R$ will refer to the set of real numbers, $\varnothing$ the empty set, and the symbol, ], will indicate the completion of a proof. If $f$ and $g$ are two functions with the property that the range of $g$ is contained in the domain of $f$, then $f \circ g$ will indicate the composition of these two functions. If $A, B$, are two sets, then $\langle A, B\rangle$ will mean the set of all functions (in whatever function space is being considered) which map $A$ into $B$. If $C$ is another set, then $\langle B, C\rangle \circ\langle A, B\rangle$ will be the set of all compositions, $f \circ g$, with $f \in\langle B, C\rangle$ and $g \in\langle A, B\rangle$. It is obvious that $\langle B, C\rangle \circ\langle A, B\rangle \subset\langle A, C\rangle$.

Received by the editors September 12, 1962; and, in revised form, January 20, 1963.

(1) The results of this paper are contained in the author's doctoral dissertation, University of Michigan, 1962. The main theorem in $\$ 3$ was presented to the Society, at its New York meeting on February 21, 1962. 
1. Definitions and known results. We consider real topological vector spaces and all terms will be as defined by Bourbaki $[1 ; 2]$. In particular, we note that a tonneau is a set which is absorbant (each element of the space is contained in some scalar multiple of the set), closed, convex and equilibrant (with each $x$ in the set, the interval from $-x$ to $x$ is in the set). A bounded set is a set which is contained in some scalar multiple of each neighborhood. A topological vector space is tonnelé if each tonneau is a neighborhood of zero. A Montel space is a topological vector space which is tonnelé and in which each closed and bounded set is compact. The spaces $\mathscr{D}, \mathscr{D}^{\prime}$ of Schwartz [10] are examples of Montel spaces.

The letters $E, F, G, \cdots$ will refer to Montel spaces and $E_{0}, E_{1}, F_{0}, F_{1}, \cdots$ to sets which are the algebraic sum of a single element and a tonneau.

The vector space $\mathscr{L}(E, F)$ of all continuous linear functions from $E$ to $F$ can be made a topological vector space with several different topologies. We consider the topology of simple convergence and the topology of uniform convergence on bounded sets. For the bounded sets relative to these topologies we shall speak, in the former case, of simply-bounded sets of functions and in the latter case, of bounded sets of functions. Unless otherwise specified, the topology of $\mathscr{L}(E, F)$ will be the topology of uniform convergence on bounded sets.

If $A, B$ are topological spaces and $C$ is a subset of $\langle A, B\rangle$, then $C$ is equicontinuous if for each neighborhood, $V$, in $B$ there is a neighborhood, $U$, in $A$ such that $C \subset\langle U, V\rangle$.

It is easy to see that if $C$ is a subset of $\mathscr{L}(E, F)$ and $C$ is equicontinuous, then $C$ is bounded. It is a basic result (in fact, a generalization of the Banach-Steinhaus theorem) that if $E$ is tonnelé, then $C$ is bounded if and only if it is simply-bounded. The proof is given in [2, p. 27].

The Riemann integral of a continuous function $f$, from $R$ to a topological vector space, $E$, is defined in the usual manner and we write $\int_{1_{0}}^{t} f(t) d t$. It is easily seen that if $f$ takes its values in a convex set, then the integral is an element of a multiple of the closure of that set.

If $f: E_{0} \rightarrow F$, then Gil de Lamadrid defines the derivative, $f^{\prime}$, to be a function from $E_{0}$ to $\mathscr{L}(E, F)$ such that for each $x \in E_{0}, B$ a bounded subset of $E, V$ a tonneau in $F$, there is a scalar, $\lambda_{0}>0$, such that if $|\lambda| \leqq \lambda_{0}$, and $h \in B$, then

$$
\frac{f(x+\lambda h)-f(x)}{\lambda}-f^{\prime}(x)(h) \in V
$$

We shall use the following notation,

$$
\delta(f, x, \lambda, h)=\frac{f(x+\lambda h)-f(x)}{\lambda}-f^{\prime}(x)(h) .
$$

If we are trying to show the existence of $f^{\prime}(x)$ and, moreover, that $f^{\prime}(x)=g(x)$, then $\delta(f, x, \lambda, h)$ will be interpreted as 


$$
\frac{f(x+\lambda h)-f(x)}{\lambda}-g(x)(h) \text {. }
$$

We remark that, since $f^{\prime}: E_{0} \rightarrow \mathscr{L}(E, F)$ and $\mathscr{L}(E, F)$ has a topology (uniform convergence on bounded sets), the continuity of $f^{\prime}$ is well defined.

We will have use of the following form of the "Fundamental Theorem of Calculus," which is proved as Theorem 14 in [5].

If $f: E_{0} \rightarrow F$ is a function whose derivative exists and is continuous, and $x_{0}, x$ are in $E_{0}$, then

$$
f(x)=f\left(x_{0}\right)+\int_{0}^{1} f^{\prime}\left(x_{0}+t\left(x-x_{0}\right)\right)\left(x-x_{0}\right) d t .
$$

2. Differential calculus. In this section we extend the results of Gil de Lamadrid [5] by proving certain theorems of calculus for the derivative which he defined.

THEOREM 1. If $f: E_{0} \rightarrow F$ is a function whose derivative exists and is continuous and $f^{\prime}\left(E_{0}\right)$ is a simply-bounded subset of $\mathscr{L}(E, F)$, then $f$ is uniformly continuous on $E_{0}$.

Proof. Since $f^{\prime}\left(E_{0}\right)$ is simply-bounded and $E$ is tonnelé, $f^{\prime}\left(E_{0}\right)$ is equicontinuous. By the Fundamental Theorem of Calculus, we have the relation,

$$
f\left(x_{1}\right)-f\left(x_{2}\right)=\int_{0}^{1} f^{\prime}\left(x_{2}+t\left(x_{1}-x_{2}\right)\right)\left(x_{1}-x_{2}\right) d t .
$$

The result then follows from the equicontinuity of $f^{\prime}\left(E_{0}\right)$ and the fact that the integral over the interval $[0,1]$ of a continuous function whose values lie in a convex set is contained in a multiple of the closure of that set.

THEOREM 2. If $f: E_{0} \rightarrow F$ is a function whose derivative exists, then

$$
\lim _{\lambda \rightarrow 0} f(x+\lambda h)=f(x),
$$

and this limit is uniform with respect to $h$ in any bounded subset, $B$, of $E_{0}$.

Proof. From the definition of $f^{\prime}$, if $V$ is a tonneau in $F$ and $\lambda$ is sufficiently small, then

$$
f(x+\lambda h)-f(x) \in \lambda f^{\prime}(x)(h)+\lambda V .
$$

But $f^{\prime}(x)(B)$ is a bounded subset of $F$ and, hence, may be absorbed by any tonneau. Thus, by taking $\lambda$ perhaps even smaller we have the relation

$$
f(x+\lambda B) \in f(x)+V
$$


Theorems 1 and 2 are substitutes for the result that differentiability implies continuity, which is false. For a counterexample, we take $\mathscr{D}$ and consider the map which sends $\phi \in \mathscr{D}$ into $\phi^{2}\left(\phi^{2}(x)=[\phi(x)]^{2}\right)$ to be a function from $\mathscr{D}$, equipped with its weak topology, into $\mathscr{D}$, equipped with its strong topology. This function is not continuous. However, since $\mathscr{D}$ is reflexive, the bounded sets are the same for both topologies. Hence, the function maps bounded sets into bounded sets from which it follows that the derivative exists at the origin.

Chain rule. The next result, which is one of the most important for our purposes, is the chain rule.

THEOREM 3. If $f: F_{0} \rightarrow G$ and $g: E_{0} \rightarrow F$ are two functions such that $g$ is continuous, $g^{\prime}$ exists, $f^{\prime}$ is continuous and $g\left(E_{0}\right) \subset F$, then $(f \circ g)^{\prime}$ exists and

$$
(f \circ g)^{\prime}(x)=\left[f^{\prime}(g(x))\right] \circ g^{\prime}(x) .
$$

Proof. From the Fundamental Theorem of Calculus, it follows that

$$
\begin{aligned}
\delta(f \circ g, x, \lambda, h)= & \int_{0}^{1} f^{\prime}\left(g(x)+\lambda g^{\prime}(x)(h)+t \lambda \delta(g, x, \lambda, h)\right)(\delta(g, x, \lambda, h)) d t \\
& +\delta\left(f, g(x), \lambda, g^{\prime}(x)(h)\right) .
\end{aligned}
$$

In the integral, the argument of $f^{\prime}$ remains in a bounded subset of $F_{0}$ as $\lambda$ ranges through any interval and $h$ ranges through a bounded subset, $B$, of $E_{0}$. This follows from the fact that $g^{\prime}(x)$ maps bounded sets into bounded sets and $g$, being continuous, maps compact sets into compact sets (here we are using the fact that in a Montel space, the closure of a bounded set is compact). Since $f^{\prime}$ is continuous, its range in the integrand is thus a bounded and hence equicontinuous subset of $\mathscr{L}(F, G)$. On the other hand, since $g^{\prime}$ exists, $\lim _{\lambda \rightarrow 0} \delta(g, x, \lambda, h)=0$ uniformly w.r.t. $h$ in $B$. Therefore, it follows that the integrand, and hence the integral, converges to 0 as $\lambda \rightarrow 0$, uniformly w.r.t. $h \in B$. For the second term, we note that as $h$ ranges through $B, g^{\prime}(x)(h)$ remains in a bounded set and the result then follows from the existence of $f^{\prime}$.

Partial derivatives. If $f: E_{0} \times F_{0} \rightarrow G$, we define the partial derivative of $f$ w.r.t. $E$ to be a function, $\partial f / \partial E: E_{0} \times F_{0} \rightarrow \mathscr{L}(E, G)$, defined by the relation

$$
\frac{\partial f}{\partial E}(x, y)(h)=\lim _{\lambda \rightarrow 0} \frac{f(x+\lambda h, y)-f(x, y)}{\lambda}, \quad x \in E_{0}, y \in F_{0},
$$

where this limit (if it exists) is taken uniformly w.r.t. $h$ in each bounded subset of $E$. We define $\partial f / \partial F$ in a similar manner.

The following result relates the partial derivatives to the so-called total derivative. The proof, using techniques introduced in the previous theorems of this section, follows classical lines. It is lengthy and will not be given. 
THEOREM 4. If $f^{\prime}$ exists, then $\partial f / \partial E, \partial f / \partial F$ exist and

$$
\begin{aligned}
& \frac{\partial f}{\partial E}(x, y)(h)=f^{\prime}(x, y)(h, 0), \\
& \frac{\partial f}{\partial F}(x, y)(k)=f^{\prime}(x, y)(0, k) .
\end{aligned}
$$

Conversely, if two partials exist and either one of them is continuous, then $f^{\prime}$ exists and

$$
f^{\prime}(x, y)(h, k)=\frac{\partial f}{\partial E}(x, y)(h)+\frac{\partial f}{\partial F}(x, y)(k) .
$$

Products and reciprocals. We consider the "product" defined by the composition of two continuous linear maps whose domain and range match properly. If $f: H_{0} \rightarrow \mathscr{L}(F, G)$ and $g: H_{0} \rightarrow \mathscr{L}(E, F)$, then we define $f \times g: H_{0} \rightarrow \mathscr{L}(E, G)$ by setting $f \times g(x)=[f(x)] \circ[g(x)]$ for all $x \in H_{0}$. If $f^{\prime}, g^{\prime}$ exist, then we define $f^{\prime} \times g: H_{0} \rightarrow \mathscr{L}(H, \mathscr{L}(E, G))$ and $f \times g^{\prime}: H_{0} \rightarrow \mathscr{L}(H, \mathscr{L}(E, G))$ by the equations

$$
\begin{aligned}
& f^{\prime} \times g(x)(h)=\left[f^{\prime}(x)(h)\right] \circ g(x), \\
& f \times g^{\prime}(x)(h)=[f(x)] \circ\left[g^{\prime}(x)(h)\right],
\end{aligned}
$$

where $x \in H_{0}, h \in H$. When there is no fear of ambiguity, the square brackets will be dropped. The inconsistency of the above notations is perhaps excused by the simplicity of the resulting product rule.

THEOREM 5. If $f: H_{0} \rightarrow \mathscr{L}(F, G), g: H_{0} \rightarrow \mathscr{L}(E, F)$ are two functions whose derivatives exist and $g$ is continuous, then $(f \times g)^{\prime}$ exists and is given by the equation

$$
(f \times g)^{\prime}=f^{\prime} \times g+f \times g^{\prime} .
$$

Proof. It is clear from the definitions that the desired equality makes sense from the point of view of the domain and range of the maps. After considerable linear manipulation, one can write,

$$
\begin{aligned}
\delta(f \times g, x, \lambda, h)= & {[\delta(f, x, \lambda, h)] \circ g(x)+f(x) \circ[\delta(g, x, \lambda, h)] } \\
& +[\delta(f, x, \lambda, h)] \circ[g(x+\lambda h)-g(x)] .
\end{aligned}
$$

To consider convergence, we recall that we are in the topology of $\mathscr{L}(E, G)$. A typical neighborhood of 0 in $\mathscr{L}(E, G)$ is $\langle B, W\rangle$ where $B$ is a convex bounded set in $E$ and $W$ is a tonneau in $G$. Since $g(x)$ is linear and continuous, there is a bounded set, $B_{0}$, in $F$ such that $g(x) \in\left\langle B, B_{0}\right\rangle$. We take $\lambda$ sufficiently small so that $\delta(f, x, \lambda, h) \in\left\langle B_{0}, W\right\rangle$, where $h$ ranges through some bounded set in $H_{0}$. Hence, we have the relation

$$
[\delta(f, x, \lambda, h)] \circ g(x) \in\left\langle B_{0}, W\right\rangle \circ\left\langle B, B_{0}\right\rangle \subset\langle B, W\rangle .
$$


From the continuity of $f(x)$, we can find a tonneau, $V$, in $F$ such that $f(x) \in\langle V, W\rangle$ Then we take $\lambda$ sufficiently small such that $\delta(g, x, \lambda, h) \in\langle B, V\rangle$, to obtain that

$$
f(x) \circ[\delta(g, x, \lambda, h)] \in\langle V, W\rangle \circ\langle B, V\rangle \subset\langle B, W\rangle .
$$

Finally, since $H$ is a Montel space and $g$ is continuous, $g(x+\lambda h)-g(x)$ remains in a bounded subset of $\mathscr{L}(E, F)$ as $h$ ranges through a bounded set and $\lambda$ ranges through an interval. Hence, there is a bounded set, $B_{1}$, in $F$ such that $g(x+\lambda h)$ $-g(x) \in\left\langle B, B_{1}\right\rangle$. Replacing $B_{0}$ by $B_{1}$ in the argument leading to Equation (1), we see that

$$
[\delta(f, x, \lambda, h)] \circ[g(x+\lambda h)-g(x)] \in\left\langle B_{1}, W\right\rangle \circ\left\langle B, B_{1}\right\rangle \subset\langle B, W\rangle .
$$

Adding (1), (2), (3) we see that for $h$ in a preassigned bounded subset of $H$ and $\lambda$ in a sufficiently small interval,

$$
\delta(f \times g, x, \lambda, h) \in\langle 3 B, W\rangle .
$$

CoRollary. If $f: H_{0} \rightarrow \mathscr{L}(F, G), g: H_{0} \rightarrow F$ are two functions whose derivatives exist and $g$ is continuous, then the function $f g: H_{0} \rightarrow G$ defined by $f g(x)=f(x)(g(x))$ is differentiable and

$$
(f g)^{\prime}(x)(h)=\left[f^{\prime}(x)(h)\right] \circ g(x)+f(x) \circ\left[g^{\prime}(x)(h)\right] .
$$

Proof. We take $E$ to be the set of reals and make the identifications, $\mathscr{L}(E, F)=F$ $\mathscr{L}(E, G)=G$. Theorem 5 then gives the desired result.

If $f: E_{0} \rightarrow \mathscr{L}(F, F)$ and the range of $f$ consists of invertible elements of $\mathscr{L}(F, F)$ then we define the reciprocal, $f^{-1}: E_{0} \rightarrow \mathscr{L}(F, F)$, by setting $f^{-I}(x)=(f(x))^{-1}$. The following result on derivatives of reciprocals is analogous to the classical result on differentiation of matrices of functions.

THEOREM 6. If $f: E_{0} \rightarrow \mathscr{L}(F, F)$ is a differentiable function such that $f^{-I}$ exists and is continuous, then $\left(f^{-I}\right)^{\prime}$ exists and

$$
\left(f^{-I}\right)^{\prime}=-f^{-I} \times f^{\prime} \times f^{-I} .
$$

Proof. We have, after some manipulation, the equation

$$
\begin{aligned}
\delta\left(f^{-I}, x, \lambda, h\right)= & -\left[f^{-I}(x)\right] \circ[\delta(f, x, \lambda, h)] \circ\left[f^{-I}(x)\right] \\
& -\left[f^{-I}(x+\lambda h)-f^{-I}(x)\right] \circ\left[f^{\prime}(x)(h)\right] \circ\left[f^{-I}(x)\right] \\
& -\left[f^{-I}(x+\lambda h)-f^{-I}(x)\right] \circ[\delta(f, x, \lambda, h)] \circ\left[f^{-I}(x)\right]
\end{aligned}
$$

Each of these three terms can then be shown to converge to 0 uniformly w.r.t. $h$ in a bounded set as $\lambda$ goes to zero, using techniques similar to those in the previous theorem. 
3. Perfect differentials. In 1933, Kerner [8] showed that in a Banach space, a first order differential form is a perfect differential if and only if the derivative of the form is symmetric. In this section we extend this result to Montel spaces. We begin with two calculus type lemmas of a more special nature than the results of $\S 2$.

LEMMA 1. If $g: I \times E_{0} \rightarrow F$ (where $I$ is the interval $[0,1]$ ) is a differentiable function which is continuous on each bounded subset of $I \times E_{0}$, and if we define the function, $f: E_{0} \rightarrow E$, by the equation

$$
f(x)=\int_{0}^{1} g(t, x) d t
$$

then $f^{\prime}$ exists and

$$
f^{\prime}(x)(h)=\int_{0}^{1} \frac{\partial g}{\partial E}(t, x)(h) d t .
$$

Proof. From the Fundamental Theorem of Calculus and Theorem 4 of $\S 2$, we may write

$$
\delta(f, x, \lambda, h)=\int_{0}^{1} \int_{0}^{1}\left\{\frac{\partial g}{\partial E}(t, x+\sigma \lambda h)(h)-\frac{\partial g}{\partial E}(t, x)(h)\right\} d \sigma d t .
$$

If $h$ is in a bounded set $B$, then the closure of $I \times B$ is compact so $g^{\prime}$ (and hence all partials) is uniformly continuous on $I \times B$. Also, $\lim _{\lambda \rightarrow 0} \sigma \lambda h=0$ uniformly w.r.t. $\sigma \in I, h \in B$. Thus for $\lambda$ sufficiently small, the integrand and, hence, the integral will be contained in any preassigned tonneau of $F$.

LEMMA 2. If $f: E_{0} \rightarrow F$ and $\alpha: I \rightarrow R$ are two functions whose derivatives exist and are continuous, then for each $x_{0}, x \in E_{0}$,

$$
\begin{aligned}
\alpha(1) f(x)-\alpha(0) f\left(x_{0}\right)= & \int_{0}^{1} \alpha^{\prime}(t) f\left(x_{0}+t\left(x-x_{0}\right)\right) d t \\
& +\int_{0}^{1} \alpha(t) f^{\prime}\left(x_{0}+t\left(x-x_{0}\right)\right)\left(x-x_{0}\right) d t .
\end{aligned}
$$

Proof. From the corollary to Theorem 5, with $F, G, H, g$ replaced by $R, F, E, \alpha$, respectively, and identifying $\mathscr{L}(R, F)$ with $F, f \alpha$ the ordinary scalar multiplication $\alpha f$, it follows that

$$
(\alpha f)^{\prime}(x)=\alpha(x) f^{\prime}(x)+\alpha^{\prime}(x) f(x) .
$$

Applying the Fundamental Theorem of Calculus, we have the desired result.

If $T: E_{0} \rightarrow \mathscr{L}(E, F)$ and $h \in E$, we define the function $T_{h}: E_{0} \rightarrow F$, by $T_{h}(x)=T(x)(h)$. We may now prove the main result of this section which shows 
that the existence of an anti-derivative is equivalent to the symmetry of the derivative.

THeOREM 7. If $T: E_{0} \rightarrow \mathscr{L}(E, F)$ such that $T^{\prime}$ exists and is continuous, then there exists $f: E_{0} \rightarrow F$ such that $f^{\prime}=T$, if and only if for all $x \in E_{0}, h, k \in E$, we have,

$$
T^{\prime}(x)(h)(k)=T^{\prime}(x)(k)(h) .
$$

Proof. First we shall assume (4) and construct $f$. Let $x_{0} \in E_{0}$. Since $E_{0}$ is convex, if $t \in I$, the vector $x_{0}+t\left(x-x_{0}\right)$ will always be in the domain of $T$. Let the function $g: I \times E_{0} \rightarrow F$ be defined by

$$
g(t, x)=T\left(x_{0}+t\left(x-x_{0}\right)\right)\left(x-x_{0}\right) .
$$

From our calculus, we can differentiate $g$ and obtain that

$$
g^{\prime}(t, x)(r, h)=T^{\prime}\left(x_{0}+t\left(x-x_{0}\right)\right)\left(t h+r\left(x-x_{0}\right)\right)\left(x-x_{0}\right)+T\left(x_{0}+t\left(x-x_{0}\right)\right)(h) .
$$

We define the function $f: E_{0} \rightarrow F$ by the equation,

$$
f(x)=\int_{0}^{1} g(t, x) d t .
$$

It follows from Lemma 1 and Theorem 4 that

$$
\begin{aligned}
f^{\prime}(x)(h) & =\int_{0}^{1} \frac{\partial g}{\partial E}(t, x)(h) d t \\
& =\int_{0}^{1}\left[t T^{\prime}\left(x_{0}+t\left(x-x_{0}\right)\right)(h)\left(x-x_{0}\right)+T\left(x_{0}+t\left(x-x_{0}\right)\right)(h)\right] d t .
\end{aligned}
$$

From the definition of $T_{h}^{\prime}$, the symmetry assumption, and the fact that uniform convergence on bounded sets implies pointwise convergence, it follows that

$$
T^{\prime}\left(x_{0}+t\left(x-x_{0}\right)\right)(h)\left(x-x_{0}\right)=T_{h}^{\prime}\left(x_{0}+t\left(x-x_{0}\right)\right)\left(x-x_{0}\right) .
$$

Hence,

$$
f^{\prime}(x)(h)=\int_{0}^{1}\left[t T_{h}^{\prime}\left(x_{0}+t\left(x-x_{0}\right)\right)\left(x-x_{0}\right)+T_{h}\left(x_{0}+t\left(x-x_{0}\right)\right)\right] d t .
$$

Applying Lemmas 1 and 2, we obtain that

$$
f^{\prime}(x)(h)=T_{h}(x)=T(x)(h) .
$$

This proves that the symmetry implies the existence of an anti-derivative. To demonstrate the converse, we suppose that $f$ is an anti-derivative of $T$. Let $h, k \in E$ 
and let $u$ be a continuous linear functional on $F$. We define the function $g: I \times I \rightarrow R$ by

$$
g(s, t)=u[f(x+s h+t k)] .
$$

It is easy to see that $g$ has continuous, second order, partial derivatives so that the two mixed partials are equal. In particular, this is so for $s=t=0$. But from the chain rule it then follows that $u\left[T^{\prime}(x)(h)(k)\right]=u\left[T^{\prime}(x)(k)(h)\right]$, and since the equality holds for every continuous linear functional, $u$, on $F$, we conclude from the Hahn-Banach Theorem that $T^{\prime}(x)(h)(k)=T^{\prime}(x)(k)(h)$.

4. Existence and uniqueness theorems. Let $T: E_{1} \times F_{0} \rightarrow \mathscr{L}(E, F)$, where $x_{0} \in E_{1}, y_{0} \in F_{0}$. In this section, we discuss the existence and uniqueness of a function $f: E_{0} \rightarrow F_{0}$, where $x_{0} \in E_{0} \subset E_{1}$, satisfying the following differential equation with initial condition,

$$
\begin{aligned}
& f\left(x_{0}\right)=y_{0} \\
& f^{\prime}(x)=T(x, f(x)) \quad \text { for all } x \in E_{0} .
\end{aligned}
$$

The dimension of $E$ (if it exists) will be the dimension of the problem. The first observation to be made is that by an obvious transformation, we can reduce all cases to that in which $x_{0}=y_{0}=0$, and we will only consider this case.

Dieudonné [4] has shown that if $E=R, F$ is a Banach space and $T$ is continuous, then the solution does not necessarily exist. We shall extend Dieudonné's example to the case in which $F$ is a Montel space.

Let $F=\mathscr{R}$, where $\mathscr{R}$ is the space of infinitely differentiable functions of rapid decrease on $R$ (see Schwartz [11, p. 89]). Let $E=R$. Let $\phi:[0,1] \rightarrow R$ be defined by the equation,

$$
\phi(\xi)=a \int_{0}^{\xi} \exp \left(-\frac{1}{1-(2 \eta-1)^{2}}\right) d \eta
$$

where $a$ is a number such that $\phi(1)=1$. It then follows that $\phi$ is infinitely differentiable, all derivatives of positive order vanish at 0,1 and $\phi(0)=0$. We define $T: R \times \mathscr{R} \rightarrow \mathscr{L}(R, \mathscr{R})=\mathscr{R}$ by setting, for $x \in R, y \in \mathscr{R}, \xi \in[n, n+1]$,

$$
T(x, y)(\xi)=(\sqrt{ }|y(n+1)|-\sqrt{ }|y(n)|) \phi(\xi-n)+\sqrt{ }|y(n)|+\exp \left(-\xi^{2}\right) .
$$

Note that $T$ is actually independent of $x$. It is easy to see that $T(x, y) \in \mathscr{R}$ and $T$ is continuous. Let us suppose then that there exists a function, $f$, satisfying (5) with $x_{0}=y_{0}=0$. We define $g_{\xi}: I \rightarrow R$ by $g_{\xi}(x)=f(x)(\xi)$. Then

In particular,

$$
g_{\xi}^{\prime}(x)=f^{\prime}(x)(\xi)=T(x, f(x))(\xi)
$$

$$
g_{n+1}^{\prime}(x)=\sqrt{ }|f(x)(n+1)|+\exp \left(-\xi^{2}\right) \geqq \sqrt{ }|f(x)(n+1)|=\sqrt{ }\left|g_{n+1}(x)\right| \text {. }
$$


Thus, $g_{n+1}(x)$ is larger than the function which vanishes at 0 and satisfies

$$
\bar{g}_{n+1}^{\prime}(x)=\sqrt{ }\left|\bar{g}_{n+1}(x)\right| \text {. }
$$

This equation has the unique solution, $\bar{g}_{n+1}(x)=x^{2} / 4$ and hence,

$$
f(x)(n+1)=g_{n+1}(x) \geqq \frac{x^{2}}{4},
$$

which contradicts the fact that $f(x) \in \mathscr{R}$.

Hence, the continuity of $T$ is not enough. We shall show that existence can be proven if we assume that $T, T^{\prime}$ are continuous, and the range of $T$ is simplybounded. We shall give the proof separately for the cases in which $E$ is onedimensional, two-dimensional, and nondimensional. All are different and each uses the previous ones. In the latter two cases, we will also have to assume that $T^{\prime}$ satisfies a certain symmetry condition.

The one-dimensional case.

THEOREM 8. Let $E=R, E_{1}=\left[0, a_{1}\right], a_{1}>0$. Let $T: E_{1} \times F_{0} \rightarrow \mathscr{L}(E, F)=F$ be a continuous function whose range, $T\left(E_{1} \times F_{0}\right)$, is a bounded subset of $F$. Then there exists $E_{0}=\left[0, a_{0}\right], 0<a_{0} \leqq a_{1}$, and $f: E_{0} \rightarrow F$ such that $f$ is continuous and differentiable, and

$$
\begin{aligned}
f(0) & =0, \\
f^{\prime}(x) & =T(x, f(x)) .
\end{aligned}
$$

Proof. Since $T\left(E_{1} \times F_{0}\right)$ is bounded in $F$, there exists a number, $a_{0}$, in the half-open interval, $\left(0, a_{1}\right]$, such that $T\left(E_{1} \times F_{0}\right)\left(\left[0, a_{0}\right]\right) \subset F_{0}$. Thus, if $B$ is the convex and topological closure of $T\left(E_{1} \times F_{0}\right)\left(E_{0}\right)$, where $E_{0}=\left[0, a_{0}\right]$, then $E_{0} \times B$ is compact, so $T$ is uniformly continuous on $E_{0} \times B$. Let $V$ be a tonneau in $F$. Let $W$ be a tonneau in $F$, and $\delta_{1}$ a positive number with the property that if $x_{1}, x_{2} \in E_{0}, y_{1}, y_{2} \in B,\left|x_{1}-x_{2}\right|<\delta_{1}$, and $y_{1}-y_{2} \in W$, then

$$
T\left(x_{1}, y_{1}\right)-T\left(x_{2}, y_{2}\right) \in\left\langle E_{0}, V\right\rangle \text {. }
$$

Let $\delta_{2}>0$ be such that $T\left(E_{0} \times F_{0}\right)\left(\left[0, \delta_{2}\right]\right) \subset W$. Let $\delta=\min \left(\delta_{1}, \delta_{2}\right)$. From these relations it follows that if we define a function $f_{V}$ on $E_{0}$ by the equations,

$$
f_{V}(x)= \begin{cases}0 & \text { if } x=0 \\ f_{V}(k \delta)+T\left(k \delta, f_{V}(k \delta)\right)(x-k \delta) & \text { if } x \in[k \delta,(k+1) \delta] \cap E_{0}\end{cases}
$$

where $k=0,1, \cdots$, then the range of $f_{V}$ is contained in $B$. To see this we have, using induction, that,

$$
\begin{aligned}
f_{V}((k+1) \delta) & =f_{V}(k \delta)+\frac{\delta}{a_{0}} T\left(k \delta, f_{V}(k \delta)\right)\left(a_{0}\right) \in \frac{k \delta}{a_{0}}+\frac{\delta}{a_{0}} B \\
& \subset \frac{k \delta}{a_{0}} B+\frac{\delta}{a_{0}} B \subset \frac{(k+1) \delta}{a_{0}} B \subset B .
\end{aligned}
$$


Thus, $f_{V}: E_{0} \rightarrow B$. It also follows that if $x \in(k \delta,(k+1) \delta)$, then

$$
f_{V}(x)-f_{V}(k \delta)=T\left(k \delta, f_{V}(k \delta)\right)(x-k \delta) \in W .
$$

If $x$ is not an integral multiple of $\delta$, we may differentiate $f$ and it follows from (7) that

$$
f_{V}^{\prime}(x)(t)-T\left(x, f_{V}(x)\right)(t)=T\left(k \delta, f_{V}(k \delta)\right)(t)-T\left(x, f_{V}(x)\right)(t) \in V .
$$

Integrating, we obtain,

$$
f_{V}(x)-\int_{0}^{x} T\left(t, f_{V}(t)\right) d t=f_{V}(x)-\int_{0}^{1} T\left(t x, f_{V}(t x)\right)(x) d t \in V
$$

Let $U$ be a tonneau in $F$. Let $\eta>0$ be such that $T\left(E_{0} \times F_{0}\right) \subset\langle[-\eta, \eta], U\rangle$. Then if $x_{1} \leqq x_{2}, x_{1} \in[(j-1) \delta, \delta]$, and $x_{2} \in[k \delta,(k+1) \delta]$, it follows that

$$
\begin{aligned}
f_{V}\left(x_{2}\right)-f_{V}\left(x_{1}\right)= & T\left(k \delta, f_{V}(k \delta)\right)\left(x_{2}-k \delta\right)+T\left((j-1) \delta, f_{V}((j-1) \delta)\right)\left(j \hat{\partial}-x_{1}\right) \\
& +\sum_{i=j}^{k-1} T\left(i \delta, f_{V}(i \delta)\right)(\delta) \in \frac{x_{2}-k \delta}{\eta} U+\frac{j \delta-x_{1}}{\eta} U+\sum_{i=j}^{k-1} \frac{\delta}{\eta} U \\
\subset & \frac{1}{\eta}\left[x_{2}-k \delta+j \delta-x_{1}+(k-j) \delta\right] U=\frac{x_{2}-x_{1}}{\eta} U .
\end{aligned}
$$

Thus, the family $\left\{f_{V}\right\}$ is an equicontinuous subset of the space $C$ of continuous functions from $E_{0}$ to $B$, equipped with the sup topology. By Ascoli's theorem [3], $\left\{f_{V}\right\}$ is a compact subset of $C$. The filter of tonneaus $\{V\}$ induces a filter structure on $\left\{f_{V}\right\}$ and the intersection of these filters is nonempty. Hence, there exists $f$ in $C$ satisfying (8) for each $V$. This implies that

$$
f(x)=\int_{0}^{x} T(t, f(t)) d t
$$

and (6) is obtained by differentiating.

THEOREM 9. Under the conditions of Theorem 8, if $T^{\prime}$ exists and is continuous and $(\partial T / \partial F)\left(E_{0} \times B\right)(B) \subset k B$ for some scalar $k$ (where $B$ is as defined in the previous theorem), then the solution is unique.

Proof. Suppose $f, g$ are solutions of (6) which vanish at 0 . Then

$$
\begin{aligned}
f(x)-g(x) & =\int_{0}^{x}\{T(\xi, f(\xi))-T(\xi, g(\xi))\} d \xi \\
& =\int_{0}^{x} \int_{0}^{1} \frac{\partial T}{\partial F}(\xi, g(\xi)+\sigma[f(\xi)-g(\xi)])(f(\xi)-g(\xi)) d \sigma d \xi .
\end{aligned}
$$

Let $B$ be as in Theorem 8 so that the topological closure, $B_{1}$, of $B-B$ is closed, convex and bounded. From (9) we see that for $x \in E_{0}$, 


$$
f(x)-g(x) \in x B-x B \subset x B_{1} .
$$

We define the function, $\alpha: E_{0} \rightarrow E$, by $\alpha(x)=\inf \left\{\lambda \mid \lambda \geqq 0, f(x)-g(x) \in \lambda B_{1}\right\}$. It follows from (10) that $\alpha$ is continuous. Also, if $f(x)-g(x) \neq 0$, by (11) we have $\alpha(x)>0$. Hence, $\alpha(x)=0$ if and only if $f(x)=g(x)$. Let $x_{1}$ be the largest number such that $\alpha\left(x_{1}\right)$ vanishes. Suppose $x_{1}<a_{0}$. Let $x_{2} \in\left[x_{1}, a_{0}\right]$ such that $x_{2}-x_{1}<1 / 2 k$ and let $x_{3} \in\left[x_{1}, x_{2}\right]$ so that $x_{3}$ is the number at which $\alpha$ attains its maximum over $\left[0, x_{2}\right]$. Hence, if $x \in\left[0, x_{2}\right]$, then $f(x)-g(x) \in \alpha\left(x_{3}\right) B_{1}$. We define the function $h: E_{0} \rightarrow F$ by the equation,

$$
h(\xi)=\int_{0}^{1} \frac{\partial T}{\partial F}(\xi, g(\xi)+\sigma[f(\xi)-g(\xi)])\left(\frac{f(\xi)-g(\xi)}{\alpha\left(x_{3}\right)}\right) d \sigma .
$$

It follows from the last equation that $h$ is continuous and $h(\xi) \in 2 k B_{1}$. Further, if $x \in\left[0, x_{2}\right]$, by $(10)$

$$
\int_{0}^{x} h(\xi) d \xi=\frac{f(x)-g(x)}{\alpha\left(x_{3}\right)} \in B_{1} .
$$

But $h(x) \in 2 k B_{1}$ for all $x \in\left[x_{0}, x_{2}\right]$. Thus, since $f(x)-g(x)$ vanishes on $\left[0, x_{1}\right]$, we have the equation

$$
f\left(x_{3}\right)-g\left(x_{3}\right)=\alpha\left(x_{3}\right) \int_{0}^{x_{3}} h(\xi) d \xi=\alpha\left(x_{3}\right) \int_{x_{1}}^{x_{3}} h(\xi) d \xi \in \alpha\left(x_{3}\right)\left(x_{3}-x_{1}\right) 2 k B_{1} .
$$

But $x_{3}-x_{1}<1 / 2 k$ and this contradicts the definition of $\alpha\left(x_{3}\right)$. Thus $x_{1}=a_{0}$ and $f(x)=g(x)$ for all $x \in E_{0}$.

It should be noted that the above proof is clearly valid if, instead of (6) we only assume (9).

As an example of the existence theorem, we take $E_{1}=[0,1], F=\mathscr{D}$ and in the notation of $[10], F_{0}=V\left(\left\{m_{V}\right\},\left\{\varepsilon_{V}\right\}\right)$ with $m_{0} \geqq 1, \varepsilon_{0} \leqq 1$. Let $K: E_{1} \times R \times E_{1} \rightarrow R$ be a function such that if $x, \sigma$ is fixed, then $K(x, t, \sigma)$, as a function of $t$, is an element of $\mathscr{D}$ and as $x, \sigma$ run through $E_{1}$, these elements of $\mathscr{D}$ form a bounded subset of $\mathscr{D}$. Further, we assume that each partial of $K$ with respect to $t$ is continuous in $x, \sigma$, uniformly with respect to $t$, and that $K(x, t, 0)=0$ for all $x \in E_{1}, t \in R$. More specifically, we might take

$$
K(x, t, \sigma)= \begin{cases}\sigma \exp \left(-\frac{1}{1-[(2+x)(2+\sigma) t]^{2}}\right) & \text { if }|(2+x)(2+\sigma) t| \leqq 1, \\ 0 & \text { if }|(2+x)(2+\sigma) t| \geqq 1\end{cases}
$$

Then we define $T: E_{1} \times F_{0} \rightarrow F$ by the equation

$$
T(x, y)(t)=\int_{-\infty}^{\infty} K\left(x, t, y^{\prime}(\xi)\right) d \xi .
$$


The conditions of Theorem 8 are satisfied, $a_{0}=1$ and we may assert the existence of a function $f: E_{1} \times R \rightarrow R$ such that

$$
\frac{\partial f}{\partial x}(x, t)=\int_{-\infty}^{\infty} K\left(x, t, \frac{\partial f}{\partial t}(x, \xi)\right) d \xi .
$$

The two-dimensional case. Because of the construction which we shall use in proving the main theorem of this section, it is necessary to have slight generalizations of some of the results of $\$ 2$. The proofs in most cases are similar and will only be sketched. A function defined on an interval is said to be continuous except for jumps on a set $S$, where $S$ is a finite subset of the interval, if the function is continuous on each interval whose interior is disjoint from $S$. We define the integral of such a function to be the sum of the integrals over the intervals of continuity. If the function is defined over a two-dimensional square, we permit the set of discontinuities to be of the form

$$
S=\left\{(t, x) / t=y_{i}, i=1, \cdots, m \text { or } t x=x_{j}, j=1, \cdots, m\right\}
$$

and continuity and integration are defined in the same way. We take $I$ to be the unit interval in $R$.

In the usual way, one can differentiate the integral by differentiating each term of a finite sum to obtain the following result.

LEMMA 3. Let the function $f: I \rightarrow F$ be continuous except for jumps on $S$ and define the function $g: I \rightarrow F$ by $g(x)=\int_{0}^{x} f(t) d t$. Then $g^{\prime}$ exists and is continuous except for jumps on $S$, and if $x \notin S$, then $g^{\prime}=f$.

Next, the classical result, along with the Hahn-Banach theorem leads to

LEMmA 4. If $f^{\prime}$ exists on $I \sim S$ and vanishes thereon, then $f$ is constant on each interval whose interior is disjoint from $S$.

If $f^{\prime}$ has jumps and we integrate the derivative of $f$, we do not necessarily get $f$ because we must jump at each element of $S$. However, if we assume that $f$ is continuous, then there are no jumps. The result is,

LEMMA 5. If $f$ is continuous and $f^{\prime}$ is continuous except for jumps on $S$, then

$$
f(x)=f(0)+\int_{0}^{x} f^{\prime}(t) d t .
$$

Now we consider the case in which $f$ is defined on $I \times I$ and the partials of $f$ have jumps. We integrate with respect to one variable and differentiate with respect to the other. Here we must pay close attention to the jumps.

LEMMA 6. Let $f: I \times I \rightarrow F$ be a continuous function whose partials are continuous except for jumps on $S$ as defined by (13). Let the function, $g: I \rightarrow F$, 
be defined by $g(x)=\int_{0}^{1} f(t, x) d t$. Then $g$ is continuous and $g^{\prime}$ is continuous except for jumps on the set $S_{0}=\left\{x_{1}, \cdots, x_{n}\right\}$, and if $x \notin S_{0}$,

$$
g^{\prime}(x)=\int_{0}^{1} \frac{\partial f}{\partial t}(t, x) d t .
$$

Proof. For each $x \in I$ we let $t_{v}(x)=y_{i}$, or if $x \in\left[0, x_{j}\right]$, then we let $t_{v}(x)=1$, or if $x \in\left[x_{j}, 1\right], t_{v}(x)=x_{j} / x$. Thus $v$ runs through a set of integers which is at most $m+n+1$ (duplicates are dropped). For each $v$, we define the function $g_{v}: I \rightarrow F$ by

$$
g_{v}(x)=\int_{t .(x)}^{t . .+1(x)} f(t, x) d t .
$$

Using the previous lemmas one can compute the derivative of $g_{v}$. These computations are lengthy but straightforward and will not be reproduced here. The result is that if $x \notin S_{0}$, then

$$
g_{v}^{\prime}(x)=\int_{t_{v}(x)}^{t_{v+1}(x)} \frac{\partial f}{\partial x}(t, x) d t+t_{v+1}^{\prime}(x) f\left(t_{v+1}(x), x\right)-t_{v}^{\prime}(x) f\left(t_{v}(x), x\right) .
$$

However, by definition, $g(x)=\Sigma_{v} g_{v}(x)$ so,

$$
g^{\prime}(x)=\sum_{v} g_{v}^{\prime}(x)=\int_{0}^{1} \frac{\partial f}{\partial x}(t, x) d t
$$

because the remaining terms telescope, the first and last being zero.

Next we have two more lemmas which generalize results of $\$ 2$. The proofs are trivial.

LEMMA 7. If $f: I \rightarrow F$ is a function whose derivative is continuous, except for jumps on $S$ and $\alpha: I \rightarrow I$ is a function whose derivative is continuous, then $f \circ \alpha$ and $\alpha f$ have derivatives which are continuous except for jumps on $S$ and

$$
(f \circ \alpha)^{\prime}(t)=f^{\prime}(\alpha(t)) \alpha^{\prime}(t) ; \quad(\alpha f)^{\prime}(t)=\alpha^{\prime}(t) f(t)+\alpha(t) f^{\prime}(t) .
$$

LEMMA 8. If $f: I \rightarrow f$ and $\alpha: I \rightarrow I$ are two functions such that $f^{\prime}$ is continuous except for jumps on $S_{1}$, and $\alpha$ is continuous except for jumps on $S_{2}$, then

$$
\int_{0}^{1} \alpha(t) f(t) d t=\left(\int_{0}^{1} \alpha(t) d t\right) f(1)-\int_{0}^{1} \int_{0}^{t} \alpha(\tau) f^{\prime}(t) d \tau d t .
$$

The next lemma may now be proven as was Theorem 7, replacing an equality with an inequality.

LEMMA 9. Let $S=\left\{(x, y) \in I \times I / x=x_{i}, i=1, \cdots, n\right.$ or $\left.y=y_{j}, j=1, \cdots, n\right\}$. Let $P, Q: I \times I \rightarrow F$ be two functions which are continuous except for jumps on $S$. Let $f: I \times I \rightarrow F$ be defined by 


$$
f(x, y)=\int_{0}^{1}\{x P(t x, t y)+y Q(t x, t y)\} .
$$

For each $x, y$, let $A_{x, y}$ be a closed, convex, equilibrant subset of $F$ with the property that for all $t$ such that $(t x, t y) \notin S$,

$$
\frac{\partial Q}{\partial x}(t x, t y)-\frac{\partial P}{\partial y}(t x, t y) \in A_{x, y}
$$

Then $f$ is continuous, $f^{\prime}$ is continuous except for jumps on $S$ and

$$
\begin{aligned}
& \frac{\partial f}{\partial x}(x, y)-P(x, y) \in \frac{y}{2} A_{x, y}, \\
& \frac{\partial f}{\partial y}(x, y)-P(x, y) \in \frac{x}{2} A_{x, y}
\end{aligned}
$$

We are now ready for the main theorem.

THEOREM 10. Let $E=R \times R, \quad I_{x}=[0, a], \quad I_{y}=[0, b], \quad E_{1}=I_{x} \times I_{y}$, $T: E_{1} \times F_{0} \rightarrow \mathscr{L}(E, F)$ such that $T, T^{\prime}$ are continuous and if $x \in I_{x}, y \in I_{y}, z \in F_{0}$, $h \in E, k \in E$, then

$$
\frac{\partial T}{\partial E}(x, y, z)(h)(k)+\frac{\partial T}{\partial F}(x, y, z)(T(x, y, z)(h))(k)
$$

is symmetric in $h, k$. Assume further that $T\left(E_{1} \times F_{0}\right)$ is simply-bounded and for each $(x, y) \in E_{1}$, the function $T_{x, y}: I \times F_{0} \rightarrow \mathscr{L}(R, F)$ defined by $T_{x, y}(t, z)(s)=T(t x, t y, z)(s x, s y)$ satisfies the conditions of Theorem 9. Then there exists an interval, $I_{0}=\left[0, a_{0}\right] \subset I_{x} \cap I_{y}$, and $a$ unique funcion $f: I_{0} \times I_{0} \rightarrow F_{0}$ such that $f(0,0)=0$, and

$$
f^{\prime}(x, y)=T(x, y, f(x, y)) .
$$

Proof. For convenience, we define the functions $P, Q: E_{1} \times F_{0} \rightarrow F$ by setting $P(x, y, z)=T(x, y, z)(1,0)$ and $Q(x, y, z)=T(x, y, z)(0,1)$. Then from the symmetry condition which is assumed, along with the results on partial differentation it follows that

$$
\frac{\partial P}{\partial y}(x, y, z)+\frac{\partial P}{\partial F}(x, y, z)(Q(x, y, z))=\frac{\partial Q}{\partial x_{1}}(x, y, z)+\frac{\partial Q}{\partial F}(x, y, z)(P(x, y, z)) .
$$

Since the range of $T$ is simply-bounded and $E$ is tonnelé, the range of $T$ is equicontinuous so there exists a number, $M>0$, such that if $|s|,|t| \leqq M$, then $T\left(E_{1} \times F_{0}\right)(s, t) \subset F_{0}$. We define $a_{0}=\min \left(\frac{1}{2} M, a, b\right)$. We take a tonneau, $V$, in $F$ and proceed as in Theorem 8. The square $E_{0}=I_{0} \times I_{0}$ is partitioned into sufficiently small subsquares. We call the partition $\pi=\pi(V)$, and the vertices 
$\left(x_{i}, y_{i}\right)$ where $i, j=0,1, \cdots, n+1$. If $(x, y) \in E_{0}$, we define $[x]=x_{i},[y]=y_{i}$, when $x \in\left[x_{i}, x_{i+1}\right)$ and $y \in\left[y_{i}, y_{i+1}\right)$. Then we define $f_{\pi}$ on $E_{0}$ by setting $f_{\pi}(0,0)=0$ and

$$
f_{\pi}(x, y)=f_{\pi}([x],[y])+T\left([x],[y], f_{\pi}([x],[y])\right)(x-[x], y-[y]) .
$$

Using the same arguments as in Theorem 8 we see that $f_{\pi}: E_{0} \rightarrow F_{0} \cap B$, where $B$ (a bounded set), is the convex and topological closure of $T\left(E_{1} \times F_{0}\right)\left(E_{1}\right)$, and we obtain the following relationships,

(15) $f_{\pi}(x, y)-f_{\pi}\left(x^{*}, y^{*}\right) \in\left(\left|x-x^{*}\right|+\left|y-y^{*}\right|\right) B$, for all $(x, y),\left(x^{*}, y^{*}\right) \in E_{0}$.

$$
f_{\pi}^{\prime}(x, y)(s, t)=P\left(x, y, f_{\pi}(x, y)\right) s+Q\left(x, y, f_{\pi}(x, y)\right) t+\Delta P s+\Delta Q t,
$$

where $\Delta P, \Delta Q \in \frac{1}{4} V$ and $x, y$ is not one of the mesh points of the partition. Now we define the functions $\tilde{P}, \tilde{Q}, \tilde{f}_{\pi}: E_{0} \rightarrow F$ by

$$
\begin{aligned}
& \tilde{P}(x, y)=P\left(x, y, f_{\pi}(x, y)\right), \quad \tilde{Q}(x, y)=Q\left(x, y, f_{\pi}(x, y)\right), \\
& f_{\pi}(x, y)=\int_{0}^{1}\{x \widetilde{P}(t x, t y)+y \widetilde{Q}(t x, t y)\} d t .
\end{aligned}
$$

We may then compute, if $(t x, t y)$ is not one of the mesh points, from (16), (14)

$$
\begin{aligned}
\frac{\partial \tilde{P}}{\partial y}(t x, t y)= & t \frac{\partial P}{\partial y}\left(t x, t y, f_{\pi}(t x, t y)\right)+t \frac{\partial P}{\partial F}\left(t x, t y, f_{\pi}(t x, t y)\right)\left(\frac{\partial f_{\pi}}{\partial y}(t x, t y)\right) \\
= & t \frac{\partial P}{\partial y}\left(t x, t y, f_{\pi}(t x, t y)\right)+t \frac{\partial P}{\partial F}\left(t x, t y, f_{\pi}(t x, t y)\right)\left(Q\left(t x, t y, f_{\pi}(t x, t y)\right)\right) \\
& +t \frac{\partial P}{\partial F}\left(t x, t y, f_{\pi}(t x, t y)\right)(\Delta Q) \\
= & t\left\{\frac{\partial Q}{\partial x}\left(t x, t y, f_{\pi}(t x, t y)\right)+\frac{\partial Q}{\partial F}\left(t x, t y, f_{\pi}(t x, t y)\right)\left(P\left(t x, t y, f_{\pi}(t x, t y)\right)\right)\right\} \\
& +t \frac{\partial P}{\partial F}\left(t x, t y, f_{\pi}(t x, t y)\right)(\Delta Q) \\
= & \frac{\partial \widetilde{Q}}{\partial x}(t x, t y)+t \frac{\partial P}{\partial F}\left(t x, t y, f_{\pi}(t x, t y)\right)(\Delta Q) \\
& -\frac{\partial Q}{\partial F}\left(t x, t y, f_{\pi}(t x, t y)\right)(\Delta P) \\
& \in \frac{\partial \tilde{Q}}{\partial x}(t x, t y)+\frac{1}{2} V .
\end{aligned}
$$


The last equation imposes an additional condition on the mesh size. This can be obtained from the uniform continuity of $T$ on $E_{0} \times B$.

Hence, by Lemma $9, f_{\pi}$ is continuous, $f_{\pi}^{\prime}$ is continuous except for jumps at the mesh points, and

$$
\frac{\partial \tilde{f}_{\pi}}{\partial x}(x, y)-\tilde{P}(x, y) \in \frac{1}{4} V, \frac{\partial \tilde{f}_{\pi}}{\partial y}(x, y)-\tilde{Q}(x, y) \in \frac{1}{4} V .
$$

From (16), (17) it follows that

$$
\frac{\partial f_{\pi}}{\partial x}(x, y)-\frac{\partial \tilde{f}_{\pi}}{\partial x}(x, y) \leq \frac{1}{2} V \quad \text { and } \quad \frac{\partial f_{\pi}}{\partial y}(x, y)-\frac{\partial \tilde{f}_{\pi}}{\partial y}(x, y) \in \frac{1}{2} V
$$

Hence, by Lemma $5, f_{\pi}(x, y)-f_{\pi}(x, y) \in V$. Thus,

$$
f_{\pi}(x, y)-\int_{0}^{1} T\left(t x, t y, f_{\pi}(t x, t y)\right)(x, y) d t \in V .
$$

It then follows from Ascoli's theorem and (15) that there exists a function $f: E_{0} \rightarrow F$ such that $f(0,0)=0$ and,

$$
f(x, y)=\int_{0}^{1} T(t x, t y, f(t x, t y))(x, y) d t .
$$

Unfortunately, (18) cannot be differentiated directly since both sides contain $f$ whose derivative is not known to exist. We proceed as follows. Fix $y \in I_{0}$ and define the function $\tilde{f}_{\pi}: I_{0} \rightarrow B$ by $\tilde{f}_{\pi}(x)=f_{\pi}(x,[y])$. Then the family $\left\{\tilde{f}_{\pi}\right\}$ is, by (15), equicontinuous in the space of continuous functions from $I_{0} \rightarrow B$. Further, if $x$ is not a mesh point, from (16), it follows that $\dot{f}_{\pi}^{\prime}(x) \in P\left(x,[y], \tilde{f}_{\pi}(x)\right)+\frac{1}{4} V$, so, by Lemma 5, we see that

$$
\tilde{f}_{\pi}(x)-\int_{0}^{x} P\left(t,[y], \tilde{f}_{\pi}(t)\right) d t \in \frac{1}{3} V .
$$

Now we apply Ascoli's theorem to the family $\left\{\bar{f}_{\pi}\right\}$, to get a function $\bar{f}: I_{0} \rightarrow F$ with the property that $\tilde{f}(x)=\int_{0}^{x} P(t, y, \tilde{f}(t)) d t$ so that

$$
f^{\prime}(x)=P(x, y, f(x)) \text {. }
$$

Now, applying Ascoli's theorem to the families $\left\{f_{\pi}\right\}$ and $\left\{\tilde{f}_{\pi}\right\}$ gives us two neighborhood bases in $F$ on which we have convergence. The intersection of these two bases is again a base and we take the following limits on the filter induced by this new base.

$$
\begin{aligned}
\tilde{f}(x)=\lim \tilde{f}_{\pi}(x)=\lim f_{\pi}(x,[y]) & =\lim f_{\pi}(x, y)+\lim \left(f_{\pi}(x,[y])-f_{\pi}(x, y)\right) \\
& =\lim f_{\pi}(x, y)=f(x, y)
\end{aligned}
$$


This result, along with (19) implies that $(\partial f / \partial x)(x, y)=P(x, y, f(x))$. In a similar manner, we show that $(\partial f / \partial y)(x, y)=Q(x, y, f(x))$. Then, Theorem 7 gives the desired result.

For the uniqueness we merely apply Theorem 9 to (18).

The nondimensional case. We can now prove the main result of this paper. $E$ is again an arbitrary Montel space.

THEOREM 11. Let $T: E_{1} \times F_{0} \rightarrow \mathscr{L}(E, F)$ be a continuous and differentiable function whose range is a simply bounded subset of $\mathscr{L}(E, F)$ and such that for all $x \in E_{1}, y \in F_{0}, h, k \in E$ the partials of $T$ are such that the expression,

$$
\frac{\partial T}{\partial E}(x, y)(h)(k)+\frac{\partial T}{\partial F}(x, y)(T(x, y)(h))(k)
$$

is symmetric in $h, k$. Assume further that for each $x \in E_{1}$, the function $T_{x}$ : $I \times F \rightarrow \mathscr{L}(R, F)$ defined by $T_{x}(t, z)(s)=T(t x, z)(s x)$ satisfies the conditions of Theorem 9.

Then there exists a tonneau $E_{0} \subset E_{1}$ and a unique function $f: E_{0} \rightarrow F$ such that $f(0)=0$ and $f^{\prime}(x)=T(x, f(x))$ for all $x \in E_{0}$.

Proof. The initial procedure is similar to that of the previous two theorems. Let

$$
E_{0}=\left[E_{1} \cap \bigcup_{E_{1} \times F_{0}}(T(x, y))^{-1}\left(F_{0}\right)\right] .
$$

Since $\mathrm{T}\left(E_{1} \times F_{0}\right)$ is an equicontinuous subset of $\mathscr{L}(E, F)$ then $E_{0}$ is a tonneau. Let $\langle B, V\rangle$ be a tonneau in $\mathscr{L}(E, F)$ where $B \subset E_{0}$. Then the closure, $T_{B}$, of $T\left(E_{0} \times F_{0}\right)(B)$ is a convex, bounded subset of $F_{0}$. We construct, on the compact set $B \times T_{B}$, an approximate solution $f_{\langle B, V\rangle}$. We choose a partition $t_{0}, \cdots, t_{n}$ of $[0,1]$ and for each $x$ on the boundary of $E_{0}$ we define, by induction, $f(0)=0$, and

$$
f(t x)=f\left(t_{i} x\right)+T\left(t_{i} x, f\left(t_{i} x\right)\right)\left(t x-t_{i} x\right), \quad t \in\left[t_{i}, t_{i+1}\right] .
$$

We have temporarily dropped the subscript $\langle B, V\rangle$ for convenience. By suitably choosing the norm, $\delta$, of the partition and a tonneau, $U \times W$, in $E \times F$, we can obtain the following results:

$$
\begin{aligned}
& x_{1}-x_{2} \in U, y_{1}-y_{2} \in W, x_{1}, x_{2} \in B, y_{1}, y_{2} \in T_{B} \text { and } h \in B \\
& \Rightarrow T\left(x_{1}, y_{1}\right)(h)-T\left(x_{2}, y_{2}\right)(h) \in V \\
& \delta B \subset U, \delta T_{B} \subset W \\
& f(t x)-f\left(t_{i} x\right) \in\left(t-t_{i}\right) T_{B} ; \\
& f(x)-\int_{0}^{1} T(t x, f(t x))(x) d t \in V \text { for all } x \in B .
\end{aligned}
$$

It also follows from (20) that $f$ is continuous. 
Since $E_{0}$ is not compact, we cannot apply Ascoli's theorem directly. Instead, for each $x \in E_{0}$ we define the functions $g_{V, x}: I \rightarrow F_{0}$ by $g_{V, x}(t)=f_{\langle B, V\rangle}(t x)$. Here, $I=[0,1]$ and we take for $B$ the intersection of $E_{0}$ and the ray through $x$. Using (21), we can meet the demands of Ascoli's theorem relative to the family of functions $\left\{g_{V \cdot x}\right\}$, with $x$ fixed, to assert the existence of a function $g_{x}: I \rightarrow F_{0}$ such that

$$
g_{x}(t)=\int_{0}^{t} T_{x}\left(\tau, g_{x}(\tau)\right) d \tau
$$

If $\alpha \in[0,1]$, then $\alpha x$ and $x$ are collinear so the same set $B$ will be used. Thus we have,

$$
g_{V, \alpha x}(t)=f_{\langle B \quad V\rangle}(t \alpha x)=g_{V x}(\alpha t) .
$$

Hence, in the limit, $g_{\alpha x}(t)=g_{x}(\alpha t)$. This permits us to define the function $f: E_{0} \rightarrow F_{0}$ by $f(x)=g_{x}(1)$ and we conclude, from (22), that

$$
f(x)=\int_{0}^{1} T_{x}\left(\tau, g_{\tau x}(1)\right) d \tau=\int_{0}^{1} T_{x}(\tau, f(\tau x)) d \tau=\int_{0}^{1} T(\tau x, f(\tau x))(x) d \tau .
$$

Thus we have solved the integral equation and it remains only for us to differentiate. To do this we must reduce the domain to $\frac{1}{4} E_{0}$. If $x_{1}, x_{2} \in \frac{1}{4} E_{0}$, we define the function, $T_{1,2}: I \times I \times F_{0} \rightarrow \mathscr{L}(R \times R, F)$, by

$$
T_{1.2}(s, t, y)(u, v)=T\left(s x_{1}+t x_{2}, y\right)\left(u x_{1}+v x_{2}\right) .
$$

The assumptions we have made regarding $T$ imply that $T_{1,2}$ satisfies the assumptions made in Theorem 10, and we may assert the existence of $I_{0}=\left[0, a_{0}\right]$, $a_{0} \leqq 1$, and $\tilde{h}: I_{0} \times I_{0} \rightarrow F_{0}$ such that $\tilde{h}(0,0)=0$ and $\tilde{h}^{\prime}(s, t)=T_{12}(s, t, \tilde{h}(s, t))$. In fact, from the proof of Theorem 10, we can actually determine that $a_{0}=1$, so $I_{0}=I$. On the other hand, if we define the function $h: I \times I \rightarrow F_{0}$ by $h(s, t)=f\left(s x_{1}+t x_{2}\right)$, it follows from (23) that

$$
h(s, t)=\int_{0}^{1} T_{1.2}(\tau s, \tau t, h(\tau s, \tau t))(s, t) d \tau .
$$

But $\tilde{h}$ also satisfies this equation, so by the uniqueness (see note at the end of Theorem 9), $h=\tilde{h}$. Therefore, $h^{\prime}$ exists and $h^{\prime}(s, t)=T_{1,2}(s, t, h(s, t))$. Therefore, if $s_{1}, s_{2}, t_{1}, t_{2} \in I$, then

$\int_{0}^{1} T_{1,2}\left(s_{1}+\sigma\left(s_{2}-s_{1}\right), t_{1}+\sigma\left(t_{2}-t_{1}\right), h\left(s_{1}+\sigma\left(s_{2}-s_{1}\right), t_{1}+\sigma\left(t_{2}-t_{1}\right)\right)\right)$

$$
\begin{aligned}
& =\int_{0}^{1} h^{\prime}\left(s_{1}+\sigma\left(s_{2}-s_{1}\right), t_{1}+\sigma\left(t_{2}-t_{1}\right)\right)\left(s_{2}-s_{1}, t_{2}-t_{1}\right) d \sigma \\
& =h\left(s_{2}, t_{2}\right)-h\left(s_{1}, t_{1}\right) .
\end{aligned}
$$


Translating back to $T$ and applying (24) three times with appropriate values of $s_{1}, s_{2}, t_{1}, t_{2}$, we see that

$\int_{0}^{1} T\left(\sigma x_{1}, f\left(\sigma x_{1}\right)\right)\left(x_{1}\right) d \sigma-\int_{0}^{1} T\left(\sigma x_{2}, f\left(\sigma x_{2}\right)\right)\left(x_{2}\right) d \sigma$

$$
\begin{gathered}
\quad+\int_{0}^{1} T\left(x_{1}+\sigma\left(x_{2}-x_{1}\right), f\left(x_{1}+\sigma\left(x_{2}-x_{1}\right)\right)\right)\left(x_{2}-x_{1}\right) d \sigma \\
=h(1,0)-h(0,0)-h(0,1)+h(0,0)+h(0,1)-h(1,0)=0 .
\end{gathered}
$$

Equation (25) states that the integral of $T$ around any triangle is zero. We may then compute, with $x_{1}=x, x_{2}=x+\lambda h$, from (23),

$$
\begin{aligned}
\frac{f(x+\lambda h)-f(x)}{\lambda}-T(x, f(x))(h) & \\
= & \int_{0}^{1}[T(x+\sigma \lambda h, f(x+\sigma \lambda h))(h)-T(x, f(x))(h)] d \sigma \\
= & \int_{0}^{1} \int_{0}^{1} T^{\prime}(x+\tau \sigma \lambda h, f(x)+\tau[f(x+\sigma \lambda h)-f(x)]) \\
& \cdot\left(\sigma \lambda h, \int_{0}^{1} T(x+\rho \sigma \lambda h, f(x+\rho \sigma \lambda h))(\sigma \lambda h) d \rho\right)(h) d \tau d \sigma .
\end{aligned}
$$

From the continuity of $T^{\prime}$ and the equicontinuity of $T\left(E_{0} \times F_{0}\right)$, the limit as $\lambda$ goes to 0 of this last expression is 0 , uniformly w.r.t. $h$ in a bounded set .Hence, $f^{\prime}(x)=T(x, f(x))$ and the existence has been proved. The uniqueness follows easily from Theorem 9 .

We close this section with an example of Theorem 11. Let $E=F=H$, the set of all complex functions which are analytic on the interior and continuous on the circumference of the unit circle. We equip $H$ with a topology by taking, as neighborhoods of 0 , the sets $V(\eta, r), \eta>0, r \in(0,1)$, and $V(\eta, r)=\{\phi \in H /|\phi(z)| \leqq \eta$, $\left|\phi^{\prime}(z)\right| \leqq \eta$, for all $z$ such that $\left.|z| \leqq r\right\}$. Now we let $E_{1}=F_{0}=V(1,1 / 2)$ and define the function $T: E_{1} \times F_{0} \rightarrow \mathscr{L}(E, F)$ by the equation,

$$
[T(x, y)(z)](\xi)=x\left(\frac{\xi}{2}\right) y\left(\frac{\xi}{2}\right) z\left(\frac{\xi}{2}\right) \text {. }
$$

The continuity of $T, T^{\prime}$ is easily verified. Further, if $z \in H, x \in E_{1}, y \in F_{0},|\xi| \leqq 1$ then

$$
[T(x, y)(z)]^{\prime}(\xi) \leqq \frac{1}{2} \sup _{|\xi| \leqq 1 / 2}\left|2 z(\xi)+z^{\prime}(\xi)\right|
$$


This shows that $T\left(E_{1} \times F_{0}\right)$ is simply-bounded. Finally,

$$
\left[\frac{\partial T}{\partial E}(x, y)(h)(k)\right](\xi)=h\left(\frac{\xi}{2}\right) y\left(\frac{\xi}{2}\right) k\left(\frac{\xi}{2}\right)
$$

and

$$
\left[\frac{\partial T}{\partial F}(x, y)(T(x, y)(k))(h)\right](\xi)=x\left(\frac{\xi}{2}\right) x\left(\frac{\xi}{2}\right) y\left(\frac{\xi}{2}\right) k\left(\frac{\xi}{2}\right) h\left(\frac{\xi}{2}\right) .
$$

Since both these expressions are symmetric in $h, k$, we have satisfied all the conditions of Theorem 11. We can compute $1 / 4 E_{0}=1 / 6 E_{1}=V(1 / 6,1 / 2)$ and we may assert the existence and uniqueness of a function $f: V(1 / 6,1 / 2) \rightarrow V(1,1 / 2)$ such that $f(0)=0$ and

$$
\left[f^{\prime}(x)(z)\right](\xi)=x\left(\frac{\xi}{2}\right)[f(x)]\left(\frac{\xi}{2}\right) z\left(\frac{\xi}{2}\right) .
$$

\section{REFERENCES}

1. N. Bourbaki, Espace vectoriels topologique, Chapters I, II, Hermann, Paris, 1953.

2. —- Espace vectoriels topologique, Chapters III, IV, V, Hermann, Paris, 1955.

3. E. A. Coddington and N. Levinson, Theory of ordinary differential equations, McGrawHill, New York, 1955.

4. J. Dieudonné, Deux exemples singuliers d'équations différentielles, Acta Sci. Math. 12 (1950), 38-40.

5. J. Gil de Lamadrid, Topology of mappings and differentiation processes, Illinois J. Math. 3 (1959). 408-420.

6. T. H. Hildebrandt and L. M. Graves, Implicit functions and their differentials in general analysis, Trans. Amer. Math. Soc. 29 (1927), 127-153.

7. D. H. Hyers, Linear topological spaces, Bull. Amer. Math. Soc. 51 (1945), 1-21.

8. M. Kerner, Die Differentiale in der Allgemeinen Analysis, Ann. of Math. 34 (1933), 546-572.

9. A. D. Michal and V. Elconin, Completely integrable differential equations in abstract spaces Acta Math. 68 (1937), 71-107.

10. L. Schwartz, Théorie des distributions, Ann. Inst. Fourier Grenoble. I, 7 (1957), 1-411.

11. — Théorie des distributions, Ann. Inst. Fourier Grenoble. II, 8 (1958), 1-209.

UNIVERSITY OF MICHIGAN, ANN ARBOR, MichigAN

UNIVERSTTY COLLEGE OF SIERRA LeONE, SirRra Leone, West Africa 